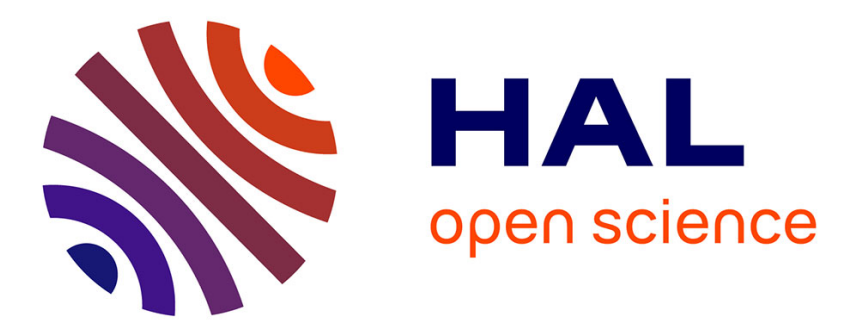

\title{
Diffraction of Gaussian beams on intracavity Bragg gratings
}

David Bitauld, Ludivine Menez, Isabelle Zaquine, Alain Maruani, Robert Frey

\section{To cite this version:}

David Bitauld, Ludivine Menez, Isabelle Zaquine, Alain Maruani, Robert Frey. Diffraction of Gaussian beams on intracavity Bragg gratings. Journal of the Optical Society of America B, 2005,22 (6), pp.1153-1160. hal-00862226

\section{HAL Id: hal-00862226 \\ https://hal-iogs.archives-ouvertes.fr/hal-00862226}

Submitted on 16 Sep 2013

HAL is a multi-disciplinary open access archive for the deposit and dissemination of scientific research documents, whether they are published or not. The documents may come from teaching and research institutions in France or abroad, or from public or private research centers.
L'archive ouverte pluridisciplinaire HAL, est destinée au dépôt et à la diffusion de documents scientifiques de niveau recherche, publiés ou non, émanant des établissements d'enseignement et de recherche français ou étrangers, des laboratoires publics ou privés. 


\title{
Diffraction of Gaussian beams on intracavity Bragg gratings
}

\author{
David Bitauld, Ludivine Menez, Isabelle Zaquine, and Alain Maruani \\ Département Traitement du Signal et de l'Image, École Nationale Supérieure des Télécommunications,
} 46 rue Barrault, 75634 Paris Cedex 13, France

\begin{abstract}
Robert Frey
Département Traitement du Signal et de l'Image, École Nationale Supérieure des Télécommunications, 46 rue Barrault, 75634 Paris Cedex 13, France, and Laboratoire Charles Fabry de l'Institut d'Optique, Centre National de la Recherche Scientifique and Université Paris XI, Centre Scientifique Paris Sud, Bt. 503, 91403 Orsay Cedex, France
\end{abstract}

Received July 30, 2004; revised manuscript received November 4, 2004; accepted December 17, 2004

\begin{abstract}
The diffraction of Gaussian beams on intracavity Bragg gratings is analyzed theoretically. For reasonable waists the associated beam divergence does not significantly influence the diffraction efficiency of such devices. Nevertheless, the tilt angle of the incident beam, imposed by the Bragg resonance condition, strongly reduces the diffraction efficiency at short grating periods. However, the angular selectivity can be maintained if the Fabry-Perot cavity is tuned to the incident beam direction, which allows the use of small-volume holograms together with a dense angular multiplex. This theoretical analysis can be applied to the optimization of the diffraction properties of Gaussian beams on any intracavity Bragg grating, which could then be used for freespace parallel signal processing. (C) 2005 Optical Society of America
\end{abstract}

OCIS codes: $090.1970,090.7330,190.4360$.

\section{INTRODUCTION}

Diffraction of light on Bragg gratings has attracted much attention, both for fundamental reasons and for applications to optical signal processing. ${ }^{1,2}$ However, because of the low refractive-index modulations or the small thicknesses of the nonlinear material used for recording these gratings, the Bragg diffraction properties of thick gratings may be insufficient for practical applications. In such a case, as shown theoretically, ${ }^{3}$ this inconvenience can be overcome by placing the sinusoidal grating in a FabryPerot cavity. More recently, and with an eye toward the design of practical devices, the Bragg criterion for such intracavity gratings ${ }^{4}$ and the influence of intracavity losses or amplification ${ }^{5}$ have been theoretically studied. Although large improvements in the diffraction properties of intracavity Bragg gratings have been obtained, ${ }^{6}$ the experimental results nevertheless remain below the theoretical predictions. This leads us to reconsider the planewave approximation of Ref. 3.

Gaussian beams have been extensively studied ${ }^{7,8}$ to account for the properties of such devices as Fabry-Perot cavities $^{9,10}$ or diffraction gratings ${ }^{11,12}$ illuminated by small diameter laser beams. In this paper we calculate the diffraction efficiency of an intracavity Bragg grating illuminated by a Gaussian beam. We show how reduced diffraction efficiency is related to the limited cross section and to the tilt angle of the incident beam, while the angular selectivity can be maintained if the incident beam is kept Fabry-Perot resonant.

Section 2 describes the model used for the intracavity Bragg diffraction of Gaussian beams and the derivation of the analytical expression of the diffraction efficiency of such beams. Section 3 discusses the profiles of the diffracted beam. Sections 4 and 5 show the influence of the waist of the Gaussian beam on the diffraction efficiency and angular selectivity, respectively.

\section{MODEL FOR THE INTRACAVITY DIFFRACTION OF GAUSSIAN BEAMS}

We use the same configuration for the Bragg device as in Ref. 5 (see Fig. 1). The Gires-Tournoi interferometer is composed of two mirrors with reflection coefficients $R_{1}$ and $R_{2} \simeq 1$ and is filled with a medium of mean refractive index $n_{0}$. The refractive-index modulation is given by $\Delta n$ $=\Delta n_{0} \cos (\mathbf{K} \cdot \mathbf{r})$, where $\Delta n_{0}$ is the maximum index modulation and $\mathbf{K}$ is the thick-grating wave vector parallel to the cavity mirrors. The $z$ axis is normal to the mirrors and the $x$ axis is the direction of the grating wave vector. Figure 1 also shows the mean directions of the wave vectors of the different waves of wavelength $\lambda$ involved in the nonlinear interaction: the incident read wave of amplitude $\mathcal{R}_{\mathrm{I}}(\mathbf{r})$ and mean wave vector $\mathbf{k}_{\text {I }}$ gives rise to intracavity forward and backward waves of respective amplitudes $\mathcal{R}_{\mathrm{F}}(\mathbf{r})$ and $\mathcal{R}_{\mathrm{B}}(\mathbf{r})$ and mean wave vectors $\mathbf{k}_{\mathrm{RF}}^{(0)}, \mathbf{k}_{\mathrm{RB}}^{(0)}$ and to the output reflected wave of amplitude $\mathcal{R}_{R}(\mathbf{r})$ and wave vector $\mathbf{k}_{\mathrm{R}}$. There is almost no transmitted wave in this GiresTournois interferometer. Because of the presence of the Bragg grating, the intracavity read waves give rise to forward and backward diffracted waves of respective amplitudes $\mathcal{S}_{\mathrm{F}}(\mathbf{r})$ and $\mathcal{S}_{\mathrm{B}}(\mathbf{r})$ and mean wave vectors $\mathbf{k}_{\mathrm{SF}}^{(0)}, \mathbf{k}_{\mathrm{SB}}^{(0)}$ and to a reflected diffracted output wave of amplitude 


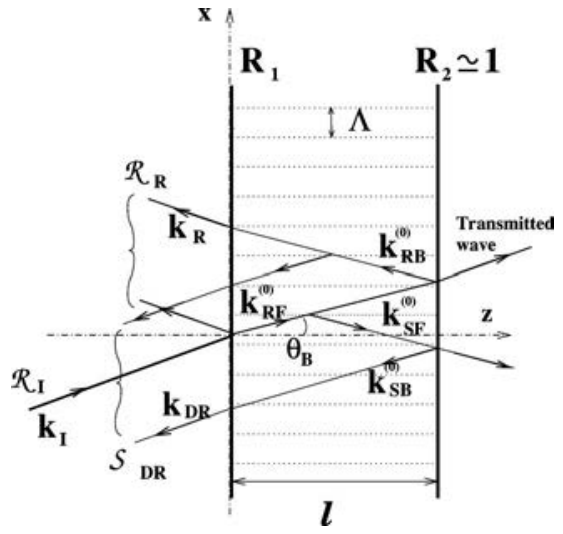

Fig. 1. Setup of the asymmetric intracavity Bragg grating of thickness $l$ and grating period $\Lambda=2 \pi / K$. The read wave of amplitude $\mathcal{R}_{I}(\mathbf{r})$ and mean wave vector $\mathbf{k}_{I}$ is incident at the Bragg angle, and the diffracted wave vector $\mathbf{k}_{\mathrm{SF}}^{(0)}$ is therefore symmetric with respect to the incident intracavity wave vector $\mathbf{k}_{\mathrm{RF}}^{(0)}$ about the $z$ axis.

$\mathcal{S}_{\mathrm{DR}}(\mathbf{r})$ and mean wave vector $\mathbf{k}_{\mathrm{DR}}$ which is counterpropagating to $\mathcal{R}_{\mathrm{I}}(\mathbf{r})$ for an incident beam set at the internal Bragg angle $\theta_{\mathrm{B}}$ (see Fig. 1).

For the calculation of the diffraction of a Gaussianintensity-profile incident beam, each of the intracavity and external fields is decomposed on the plane-wave basis. The amplitude at $z=0$ (at the first mirror) of the incident beam of peak power $P_{\mathrm{I}}$, waist $\sqrt{2} w_{0}$ (at $1 / e$ of the maximum), and polarized along the $y$ axis is

$$
\mathcal{R}_{\mathrm{I}}\left(\mathbf{r}_{0}\right)=\hat{\mathbf{y}} R_{\mathrm{I}}\left(\mathbf{r}_{0}\right) \exp \left(i \mathbf{k}_{\mathrm{I}} \cdot \mathbf{r}_{0}\right)=\hat{\mathbf{y}} R_{\mathrm{I}}\left(\mathbf{r}_{0}\right) \exp \left(i \mathbf{k}_{\mathrm{RF}}^{(0)} \cdot \mathbf{r}_{0}\right),
$$

where $\mathbf{r}_{0}=(x, y, 0), \mathbf{k}_{\mathrm{RF}}^{(0)}=k^{\prime}(\sin \theta \hat{\mathbf{x}}+\cos \theta \hat{\mathbf{z}}), \theta$ is the intracavity incidence angle, and $\hat{\mathbf{x}}, \hat{\mathbf{y}}$, and $\hat{\mathbf{z}}$ are the unit vectors of the respective $x, y$, and $z$ axes. The complex amplitude of the incident beam in the plane $z=0$ is

$$
\begin{aligned}
R_{\mathrm{I}}\left(\mathbf{r}_{0}\right)= & \frac{1}{2 \pi} \iint_{-\infty}^{+\infty} \frac{\mathrm{d} \delta k_{x}}{\cos \theta} \mathrm{d} \delta k_{y} \widetilde{R}_{\mathrm{I}}\left(\delta k_{x}, \delta k_{y}, 0\right) \\
& \times \exp \left(-i \delta k_{z} \sin \theta x\right) \exp \left[i\left(\delta k_{x} x+\delta k_{y} y\right)\right]
\end{aligned}
$$

where

$$
\widetilde{R}_{\mathrm{I}}\left(\delta k_{x}, \delta k_{y}, 0\right)=\left(\frac{2 P_{\mathrm{I}} w_{0}^{2}}{c n_{0}}\right)^{1 / 2} \exp \left[-\frac{w_{0}^{2}}{2}\left(\frac{\partial k_{x}^{2}}{\cos ^{2} \theta}+\delta k_{y}^{2}\right)\right]
$$

is the two-dimensional spatial Fourier transform at $z=0$ of the incident Gaussian beam. In Eq. (2) $\delta k_{z}$ $=\left(\delta k_{x}^{2} / \cos ^{2} \theta\right)+\delta k_{y}^{2} / 2 k^{\prime}$ ensures the conservation of the modulus $k^{\prime}=2 \pi n_{0} / \lambda$ of the wave vector to first order in $\delta k_{z}$ and allows us to take wave-front curvature into account.

The amplitudes of the forward and backward read and diffracted waves in the Fabry-Perot cavity are, respectively

$$
\mathcal{R}_{\mathrm{F}, \mathrm{B}}(\mathbf{r})=\hat{\mathbf{y}} R_{\mathrm{F}, \mathrm{B}}(\mathbf{r}) \exp \left(i \mathbf{k}_{\mathrm{RF}, \mathrm{RB}}^{(0)} \cdot \mathbf{r}\right),
$$

$$
\mathcal{S}_{\mathrm{F}, \mathrm{B}}(\mathbf{r})=\hat{\mathbf{y}} S_{\mathrm{F}, \mathrm{B}}(\mathbf{r}) \exp \left(i \mathbf{k}_{\mathrm{SF}, \mathrm{SB}}^{(0)} \cdot \mathbf{r}\right)
$$

with

$$
\begin{aligned}
& \mathbf{k}_{\mathrm{RF}}^{(0)}=k^{\prime}(\sin \theta \hat{\mathbf{x}}+\cos \theta \hat{\mathbf{z}}), \\
& \mathbf{k}_{\mathrm{RB}}^{(0)}=k^{\prime}(\sin \theta \hat{\mathbf{x}}-\cos \theta \hat{\mathbf{z}}), \\
& \mathbf{k}_{\mathrm{SF}}^{(0)}=k^{\prime}\left(\sin \theta^{\prime} \hat{\mathbf{x}}+\cos \theta^{\prime} \hat{\mathbf{z}}\right), \\
& \mathbf{k}_{\mathrm{SB}}^{(0)}=k^{\prime}\left(\sin \theta^{\prime} \hat{\mathbf{x}}-\cos \theta^{\prime} \hat{\mathbf{z}}\right),
\end{aligned}
$$

where $\theta$ and $\theta^{\prime}$ are the internal incidence and diffraction angles, respectively.

For the elementary plane waves considered in the decomposition of all fields, the Floquet theorem restricts the possible solutions in a periodic medium. Conservation of the total wave vector along the periodicity direction $x$ is required: ${ }^{13,14} \quad\left(\mathbf{k}_{\mathrm{SF}, \mathrm{SB}}^{(0)}+\delta \mathbf{k}_{\mathrm{SF}, \mathrm{SB}}\right) \cdot \hat{\mathbf{x}}=\left(\mathbf{k}_{\mathrm{RF}, \mathrm{RB}}^{(0)}+\delta \mathbf{k}_{\mathrm{RF}, \mathrm{RB}}\right) \cdot \hat{\mathbf{x}}$ $+p K$, where $p$ is any integer and where

$$
\begin{aligned}
\delta \mathbf{k}_{\mathrm{RF}}= & \left(-\delta k_{z_{\mathrm{R}}} \sin \theta+\delta k_{x}\right) \hat{\mathbf{x}}+\delta k_{y} \hat{\mathbf{y}} \\
& -\left(\delta k_{z_{\mathrm{R}}} \cos \theta+\delta k_{x} \tan \theta\right) \hat{\mathbf{z}}, \\
\delta \mathbf{k}_{\mathrm{RB}}= & -\left(\delta k_{z_{\mathrm{R}}} \sin \theta+\delta k_{x}\right) \hat{\mathbf{x}} \\
& +\delta k_{y} \hat{\mathbf{y}}+\left(-\delta k_{z_{\mathrm{R}}} \cos \theta+\delta k_{x} \tan \theta\right) \hat{\mathbf{z}},
\end{aligned}
$$

$$
\begin{aligned}
\delta \mathbf{k}_{\mathrm{SF}}= & \left(-\delta k_{z_{\mathrm{S}}} \sin \theta^{\prime}+\delta k_{x}\right) \hat{\mathbf{x}}+\delta k_{y} \hat{\mathbf{y}} \\
& +\left(\delta k_{z_{\mathrm{S}}} \cos \theta^{\prime}+\delta k_{x} \tan \theta^{\prime}\right) \hat{\mathbf{z}}, \\
\delta \mathbf{k}_{\mathrm{SB}}= & -\left(\delta k_{z_{\mathrm{S}}} \sin \theta^{\prime}+\delta k_{x}\right) \hat{\mathbf{x}}+\delta k_{y} \hat{\mathbf{y}} \\
& +\left(-\delta k_{z_{\mathrm{S}}} \cos \theta^{\prime}+\delta k_{x} \tan \theta^{\prime}\right) \hat{\mathbf{z}}
\end{aligned}
$$

are the deviations from the mean wave vectors of the intracavity beams, with $\delta k_{z_{\mathrm{R}}}=\left[\delta k_{x}^{2} / \cos ^{2} \theta+\delta k_{y}^{2}\right] / 2 k^{\prime}, \delta k_{z_{\mathrm{S}}}$ $=\left[\delta k_{x}^{2} / \cos ^{2} \theta^{\prime}+\delta k_{y}^{2}\right] / 2 k^{\prime}$ the dephasings associated with the respective plane-wave components of the Gaussian beam. In the case of a Bragg grating, only one space harmonic has to be taken into account and $p=1$. There is nevertheless a phase mismatch along the propagation direction $z$ (see Fig. 2). In the plane-wave basis, the complex amplitudes can be written
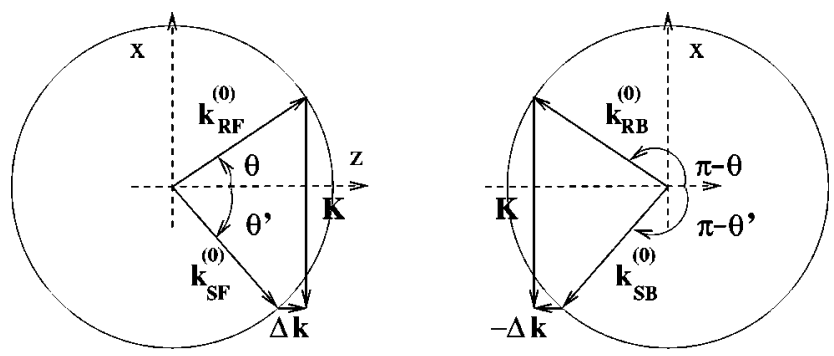

Fig. 2. Grating wave vector conservation and phase mismatch $\Delta k$ for the forward and backward read and diffracted waves when $\theta \neq \theta_{\mathrm{B}}$ and therefore $\theta^{\prime} \neq \theta$. 
$R_{\mathrm{F}}(\mathbf{r})=\frac{1}{2 \pi} \iint_{-\infty}^{+\infty} \frac{\mathrm{d} \delta k_{x}}{\cos \theta} \mathrm{d} \delta k_{y} \widetilde{R}_{\mathrm{F}}\left(\delta k_{x}, \delta k_{y}, z\right) \times \exp \left(i \delta \mathbf{k}_{\mathrm{RF}} \cdot \mathbf{r}\right)$,

$$
\begin{aligned}
S_{\mathrm{F}}(\mathbf{r})= & \frac{1}{2 \pi} \iint_{-\infty}^{+\infty} \frac{\mathrm{d} \delta k_{x}}{\cos \theta^{\prime}} \mathrm{d} \delta k_{y} \widetilde{S}_{\mathrm{F}}\left(\delta k_{x}, \delta k_{y}, z\right) \\
& \times \exp \left(i \delta \mathbf{k}_{\mathrm{SF}} \cdot \mathbf{r}\right) \exp (-i \Delta k z),
\end{aligned}
$$

$R_{\mathrm{B}}(\mathbf{r})=\frac{1}{2 \pi} \iint_{-\infty}^{+\infty} \frac{\mathrm{d} \delta k_{x}}{\cos \theta} \mathrm{d} \delta k_{y} \widetilde{R}_{\mathrm{B}}\left(\delta k_{x}, \delta k_{y}, z\right) \times \exp \left(i \delta \mathbf{k}_{\mathrm{RB}} \cdot \mathbf{r}\right)$,

$$
\begin{aligned}
S_{\mathrm{B}}(\mathbf{r})= & \frac{1}{2 \pi} \iint_{-\infty}^{+\infty} \frac{\mathrm{d} \delta k_{x}}{\cos \theta^{\prime}} \mathrm{d} \delta k_{y} \widetilde{S}_{\mathrm{B}}\left(\delta k_{x}, \delta k_{y}, z\right) \\
& \times \exp \left(i \delta \mathbf{k}_{\mathrm{SB}} \cdot \mathbf{r}\right) \exp (i \Delta k z)
\end{aligned}
$$

where $\widetilde{R}_{\mathrm{F}, \mathrm{B}}$ and $\widetilde{S}_{\mathrm{F}, \mathrm{B}}$ are the two-dimensional spatial Fourier transforms of the respective amplitudes $R_{\mathrm{F}, \mathrm{B}}$ and $S_{\mathrm{F}, \mathrm{B}}$, and $\Delta k=k^{\prime}\left(\cos \theta^{\prime}-\cos \theta\right)-\delta k_{x}\left(\tan \theta^{\prime}-\tan \theta\right)$ is the phase mismatch due to the detuning from Bragg resonance. By substituting the total intracavity field $\mathcal{E}(\mathbf{r})$ $=\mathcal{R}_{\mathrm{F}}(\mathbf{r})+\mathcal{R}_{\mathrm{B}}(\mathbf{r})+\mathcal{S}_{\mathrm{F}}(\mathbf{r})+\mathcal{S}_{\mathrm{B}}(\mathbf{r})$ into the propagation equation

$$
\Delta \mathcal{E}(\mathbf{r})+\left(k^{\prime}+i k^{\prime \prime}\right) \mathcal{E}(\mathbf{r})=\left[\frac{4 \pi^{2}}{\lambda^{2}} n_{0} \Delta n \cos (K x)\right] \mathcal{E}(\mathbf{r})
$$

and using the slowly varying amplitude approximation, ${ }^{15}$ one can derive the coupled propagation equations describing the behavior of the Fourier components $\widetilde{R}_{\mathrm{F}, \mathrm{B}}$ and $\widetilde{S}_{\mathrm{F}, \mathrm{B}}$ as

$$
\begin{array}{r}
\frac{\partial \widetilde{R}_{\mathrm{F}}}{\partial z}+\frac{k^{\prime \prime}}{\cos \theta} \widetilde{R}_{\mathrm{F}}=\frac{i \pi \Delta n}{\lambda \cos \theta^{\prime}} \widetilde{S}_{\mathrm{F}} \\
\frac{\partial \widetilde{S}_{\mathrm{F}}}{\partial z}+\left(\frac{k^{\prime \prime}}{\cos \theta^{\prime}}-i \Delta k\right) \widetilde{S}_{\mathrm{F}}=\frac{i \pi \Delta n}{\lambda \cos \theta} \widetilde{R}_{\mathrm{F}} \\
-\frac{\partial \widetilde{R}_{\mathrm{B}}}{\partial z}+\frac{k^{\prime \prime}}{\cos \theta} \widetilde{R}_{\mathrm{B}}=\frac{i \pi \Delta n}{\lambda \cos \theta^{\prime}} \widetilde{S}_{\mathrm{B}} \\
-\frac{\partial \widetilde{S}_{\mathrm{B}}}{\partial \mathrm{z}}+\left(\frac{k^{\prime \prime}}{\cos \theta^{\prime}}-i \Delta k\right) \widetilde{S}_{\mathrm{B}}=\frac{i \pi \Delta n}{\lambda \cos \theta} \widetilde{R}_{\mathrm{B}}
\end{array}
$$

Equations (19)-(22) are similar to those obtained for plane waves in a lossless material, except for the term involving $k^{\prime \prime}=\alpha / 2$, which takes into account the influence of (assumed small) absorption losses, with $\alpha$ the intensity absorption coefficient.

The resolution of this set of coupled differential equation allows us to calculate the $z$ dependence of each one of the intracavity plane waves, thus giving

$$
\widetilde{R}_{\mathrm{F}}\left(\delta k_{x}, \delta k_{y}, z\right)=A_{\mathrm{F}}^{+} \exp \left(r_{\mathrm{F}}^{+} z\right)+A_{\mathrm{F}}^{-} \exp \left(r_{\mathrm{F}}^{-} z\right)
$$

$$
\begin{aligned}
\widetilde{R}_{\mathrm{B}}\left(\delta k_{x}, \delta k_{y}, z\right)= & A_{\mathrm{B}}^{+} \exp \left(r_{\mathrm{B}}^{+} z\right)+A_{\mathrm{B}}^{-} \exp \left(r_{\mathrm{B}}^{-} z\right), \\
\widetilde{S}_{\mathrm{F}}\left(\delta k_{x}, \delta k_{y}, z\right)= & -\frac{i \lambda \cos \theta^{\prime}}{\pi \Delta n}\left[\left(r_{\mathrm{F}}^{+}+\frac{k^{\prime \prime}}{\cos \theta}\right)\right. \\
& \times A_{\mathrm{F}}^{+} \exp \left(r_{\mathrm{F}}^{+} z\right) \\
& \left.+\left(r_{\mathrm{F}}^{-}+\frac{k^{\prime \prime}}{\cos \theta}\right) A_{\mathrm{F}}^{-} \exp \left(r_{\mathrm{F}}^{-} z\right)\right], \\
\widetilde{S}_{\mathrm{B}}\left(\delta k_{x}, \delta k_{y}, z\right)= & \frac{i \lambda \cos \theta^{\prime}}{\pi \Delta n}\left[\left(r_{\mathrm{B}}^{+}-\frac{k^{\prime \prime}}{\cos \theta}\right)\right. \\
& \times A_{\mathrm{B}}^{+} \exp \left(r_{\mathrm{B}}^{+} z\right) \\
& \left.+\left(r_{\mathrm{B}}^{-}-\frac{k^{\prime \prime}}{\cos \theta}\right) A_{\mathrm{B}}^{-} \exp \left(r_{\mathrm{B}}^{-} z\right)\right],
\end{aligned}
$$

with

$$
\begin{aligned}
r_{\mathrm{F}}^{ \pm}= & -r_{\mathrm{B}}^{\mp}=-\frac{k^{\prime \prime}}{2}\left(\frac{1}{\cos \theta}+\frac{1}{\cos \theta^{\prime}}\right)+\frac{i \Delta k}{2} \pm i \sqrt{\Delta^{\prime}}, \\
\Delta^{\prime}= & {\left[\frac{\pi \Delta n}{\lambda\left(\cos \theta \cos \theta^{\prime}\right)^{1 / 2}}\right]^{2} } \\
& +\left[\frac{\Delta k}{2}+i \frac{k^{\prime \prime}}{2}\left(\frac{1}{\cos \theta}-\frac{1}{\cos \theta^{\prime}}\right)\right]^{2} .
\end{aligned}
$$

The boundary conditions for the plane waves coupled by the cavity mirrors with amplitude reflectivities $r_{1}, r_{2}$ located at $z=0$ and $z=l$ enable us to determine totally the field amplitudes in the cavity:

$$
\begin{aligned}
& \mathrm{R}_{\mathrm{F}_{\mathrm{x}} \delta \mathrm{k}_{\mathrm{y}}}(x, y, 0)=r_{1} \mathrm{R}_{B \delta \mathrm{k}_{\mathrm{x}} \delta \mathrm{k}_{\mathrm{y}}}(x, y, 0)+t_{1} \mathrm{R}_{\mathrm{I}_{\mathrm{x}} \delta \mathrm{k}_{\mathrm{y}}}(x, y, 0), \\
& \mathrm{S}_{\mathrm{F} \delta \mathrm{k}_{\mathrm{x}} \delta \mathrm{k}_{\mathrm{y}}}(x, y, 0)=r_{1} \mathrm{~S}_{\mathrm{B} \delta \mathrm{k}_{\mathrm{x}} \delta \mathrm{k}_{\mathrm{y}}}(x, y, 0), \\
& \mathrm{R}_{\mathrm{B} \delta \mathrm{k}_{\mathrm{x}} \delta \mathrm{k}_{\mathrm{y}}}(x, y, l)=r_{2} \mathrm{R}_{\mathrm{F}_{\mathrm{k}_{\mathrm{x}}} \delta \mathrm{k}_{\mathrm{y}}}(x, y, l), \\
& \mathrm{S}_{\mathrm{B} \delta \mathrm{k}_{\mathrm{x}} \delta \mathrm{k}_{\mathrm{y}}}(x, y, l)=r_{2} \mathrm{~S}_{\mathrm{F} \delta \mathrm{k}_{\mathrm{x}} \delta \mathrm{k}_{\mathrm{y}}}(x, y, l),
\end{aligned}
$$

where

$$
\begin{aligned}
& \mathrm{R}_{\mathrm{F} \delta \mathrm{k}_{\mathrm{x}}, \delta \mathrm{k}_{\mathrm{y}}}(\mathbf{r})=\hat{\mathbf{y}} \widetilde{R}_{\mathrm{F}}\left(\delta k_{x}, \delta k_{y}, z\right) \exp \left[i\left(\mathbf{k}_{\mathrm{RF}}^{(0)}+\delta \mathbf{k}_{\mathrm{RF}}\right) \cdot \mathbf{r}\right], \\
& \mathrm{R}_{\mathrm{B} \delta \mathrm{k}_{\mathrm{x}}, \delta \mathrm{k}_{\mathrm{y}}}(\mathbf{r})=\hat{\mathbf{y}} \widetilde{R}_{\mathrm{B}}\left(\delta k_{x}, \delta k_{y}, z\right) \exp \left[i\left(\mathbf{k}_{\mathrm{RB}}^{(0)}+\delta \mathbf{k}_{\mathrm{RB}}\right) \cdot \mathbf{r}\right],
\end{aligned}
$$

$$
\begin{aligned}
\mathrm{S}_{\mathrm{F} \delta \mathrm{k}_{\mathrm{x}}, \delta \mathrm{k}_{\mathrm{y}}}(\mathbf{r})= & \hat{\mathbf{y}} \widetilde{S}_{\mathrm{F}}\left(\delta k_{x}, \delta k_{y}, z\right) \exp (-i \Delta k z) \\
& \times \exp \left[i\left(\mathbf{k}_{\mathrm{SF}}^{(0)}+\delta \mathbf{k}_{\mathrm{SF}}\right) \cdot \mathbf{r}\right]
\end{aligned}
$$




$$
\begin{aligned}
\mathrm{S}_{\mathrm{B} \delta \mathrm{k}_{\mathrm{x}}, \delta \mathrm{k}_{\mathrm{y}}}(\mathbf{r})= & \hat{\mathbf{y}} \widetilde{\boldsymbol{S}}_{\mathrm{B}}\left(\delta k_{x}, \delta k_{y}, z\right) \exp (-i \Delta k z) \\
& \times \exp \left[i\left(\mathbf{k}_{\mathrm{SB}}^{(0)}+\delta \mathbf{k}_{\mathrm{SB}}\right) \cdot \mathbf{r}\right]
\end{aligned}
$$

are the plane-wave components of the intracavity beams. The values of $A_{\mathrm{F}}^{ \pm}$and $A_{\mathrm{B}}^{ \pm}$are calculated exactly by solving the system of boundary equations [Eqs. (29)-(32)] by use of the expressions of $\mathrm{R}_{\mathrm{F}, \mathrm{B} \delta \mathrm{k}_{\mathrm{x}}, \delta \mathrm{k}_{\mathrm{y}}}$ and $\mathrm{S}_{\mathrm{F}, \mathrm{B} \delta \mathrm{k}_{\mathrm{x}}, \delta \mathrm{k}_{\mathrm{y}}}$ [Eqs. (33)-(36)] where $\widetilde{R}_{\mathrm{F}, \mathrm{B}}$ and $\widetilde{S}_{\mathrm{F}, \mathrm{B}}$ have been replaced by their expressions in Eqs. (24)-(26). We obtain

$$
\begin{gathered}
A_{\mathrm{F}}^{ \pm}=\frac{ \pm i t_{1}\left(r^{\mp}+\frac{k^{\prime \prime}}{\cos \theta}\right)}{2 \sqrt{\Delta^{\prime}}\left[1-r_{1} r_{2}^{\prime} \exp \left(2 r^{ \pm} L\right)\right]} e_{\mathrm{I}}, \\
A_{\mathrm{B}}^{ \pm}=\frac{\mp i t_{1}\left(r^{ \pm}+\frac{k^{\prime \prime}}{\cos \theta}\right) r_{2}^{\prime} \exp \left(2 r^{\mp} l\right)}{2 \sqrt{\Delta^{\prime}}\left[1-r_{1} r_{2}^{\prime} \exp \left(2 r^{\mp} l\right)\right]} e_{\mathrm{I}},
\end{gathered}
$$

where $r^{ \pm}=r_{\mathrm{F}}^{ \pm}=-r_{\mathrm{B}}^{\mp}$ with reference to Eq. (27), and $r_{2}^{\prime}$ $=r_{2} \exp \left[2 i\left(k^{\prime} \cos \theta-\delta k_{x} \tan \theta\right) L\right]$.

By use of the previous results it is straightforward to calculate the transmission and reflection efficiencies and transmitted and reflected diffraction efficiencies of the device; these are defined by

$$
\begin{aligned}
\rho_{\mathrm{T}}= & \frac{c n\left(1-R_{2}\right)}{2 \pi P_{\mathrm{I}}} \iint_{-\infty}^{+\infty}\left|\widetilde{R}_{\mathrm{F}}\left(\delta k_{x}, \delta k_{y}, l\right)\right|^{2} \frac{\mathrm{d} \delta k_{x}}{\cos \theta} \mathrm{d} \delta k_{y}, \\
\rho_{\mathrm{R}}= & \frac{c n}{2 \pi P_{\mathrm{I}}} \iint_{-\infty}^{+\infty} \mid \widetilde{R}_{\mathrm{B}}\left(\delta k_{x}, \delta k_{y}, 0\right) \sqrt{1-R_{1}} \\
& -\left.\widetilde{R}_{\mathrm{I}}\left(\delta k_{x}, \delta k_{y}, 0\right) \sqrt{R_{1}}\right|^{2} \frac{\mathrm{d} \delta k_{x}}{\cos \theta} \mathrm{d} \delta k_{y}, \\
\rho_{\mathrm{DT}}= & \frac{c n\left(1-R_{2}\right)}{2 \pi P_{\mathrm{I}}} \iint_{-\infty}^{+\infty}\left|\widetilde{S}_{\mathrm{F}}\left(\delta k_{x}, \delta k_{y}, l\right)\right|^{2} \frac{\mathrm{d} \delta k_{x}}{\cos \theta} \mathrm{d} \delta k_{y}, \\
\rho_{\mathrm{DR}}= & \frac{c n\left(1-R_{1}\right)}{2 \pi P_{\mathrm{I}}} \iint_{-\infty}^{+\infty}\left|\widetilde{S}_{\mathrm{B}}\left(\delta k_{x}, \delta k_{y}, 0\right)\right|^{2} \frac{\mathrm{d} \delta k_{x}}{\cos \theta} \mathrm{d} \delta k_{y} .
\end{aligned}
$$

The double integrals in $\mathrm{d} \delta k_{x}$ and $\mathrm{d} \delta k_{y}$ appearing in the expressions of the efficiencies are calculated numerically by a Gauss integration method. The boundaries of this integration are adjusted for each calculated point to fit the domain where the function has nonnegligible values. The validity of the numerical calculations is confirmed by checking the energy conservation for a lossless medium $(\alpha=0)$ through the relation $\rho_{\mathrm{T}}+\rho_{\mathrm{R}}+\rho_{\mathrm{DT}}+\rho_{\mathrm{DR}}=1$ for various values of the parameters. All the results presented hereafter are obtained for a read beam wavelength of $1 \mu \mathrm{m}$. In Sections 3 and 4 we consider a Bragg grating placed in an asymmetric cavity $\left(R_{1}=0.80\right.$ and $\left.R_{2}=1\right)$ filled by a lossless intracavity medium. The waist $w_{0}$ and tilt angle $\theta$ of the read beam and the thickness $l$ of the FabryPerot cavity are used as parameters.
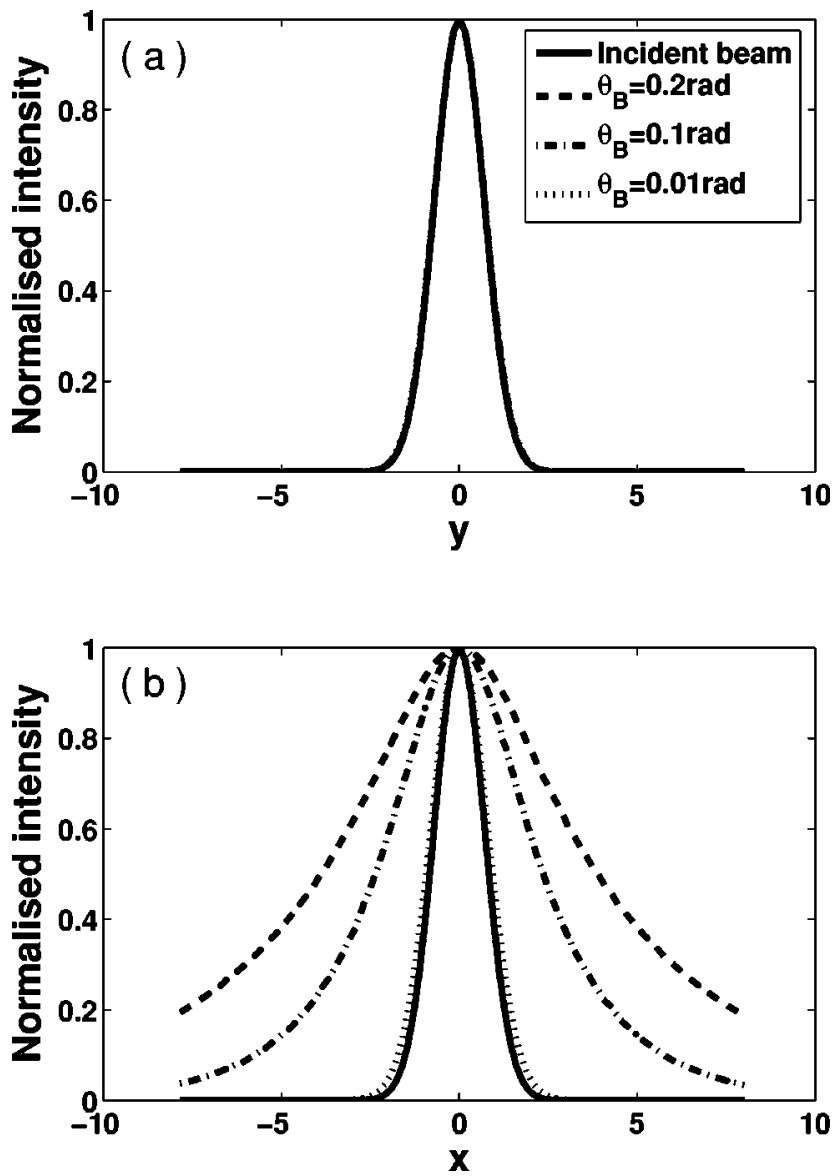

Fig. 3. Normalized (a) $y$ and (b) $x$ profiles of the diffracted beam compared with those of the incident beam at various tilt angles. The finesse of the Fabry-Perot cavity is 28, the sample thickness is $1 \mathrm{~mm}$, and the incident beam waist is $300 \mu \mathrm{m}$.

\section{3. $x$ AND $y$ PROFILES OF THE DIFFRACTED BEAM}

To analyze qualitatively the influence of diffraction and tilt angle of the incident beam, we first study the profiles of the diffracted beam. The $y$ and $x$ profiles are shown in Figs. 3(a) and 3(b), respectively, at the maximum of the field amplitude for the other coordinate, namely, $x$ and $y$. They correspond to the diffraction of a moderately focused Gaussian beam $\left(w_{0}=300 \mu \mathrm{m}\right)$ incident at the Bragg angle for three values of this angle $\left(\theta_{\mathrm{B}}=0.01,0.1\right.$, and $\left.0.2 \mathrm{rad}\right)$ on a 1-mm-thick intracavity Bragg grating. As all beam directions are located in the $x z$ plane, the diffracted beam is centered on $y=0$, and only beam divergence can modify its profile in the $y$ direction. Actually, with the parameters used in our calculations, the normalized $y$ profiles remain identical to those of the incident beam for the three values of $\theta_{\mathrm{B}}$ previously mentioned [see Fig. 3(a)]. Despite the relatively high finesse $\mathcal{F}=\pi \sqrt{r_{1} r_{2}} /\left(1-r_{1} r_{2}\right)=28$ of our cavity, this result is consistent with the low angular divergence related to the beam waist $\left(\theta_{\mathrm{D}} \sim \lambda / w_{0}=3.3 \times 10^{-3}\right)$ of the incident read beam. In the case of tighter focusing of the read beam, the widening in the $y$ direction has been verified through our calculations.

The $x$ profiles of the normalized diffracted amplitudes shown in Fig. 3(b) are strongly modified as the tilt angle increases. For low values of the tilt angle $\left(\theta_{\mathrm{B}}<0.01\right)$ they 
look almost identical to the $y$ profiles. For larger values of $\theta_{\mathrm{B}}$ the diffracted beam exhibits a spread, increasing with increasing $\theta_{\mathrm{B}}$ even though it stays centered on the incident beam. The large spread of the diffracted beam implies a reduction of the effective cavity finesse of the Fabry-Perot device. This phenomenon results in a strong decrease in the diffraction efficiency at high values of $\theta_{\mathrm{B}}$ as shown in Section 4.

\section{DIFFRACTION EFFICIENCY}

We also investigated the effect of various parameters on the diffraction efficiency of the Gaussian beam on the intracavity Bragg device. The influence of tilt angle $\theta_{\mathrm{B}}$, average thickness $l_{0}$ of the cavity, and incident beam waist $w_{0}$ are presented in Figs. 4(a)-4(c). On all these curves every data point corresponds to a resonance length for the Fabry-Perot device and to an adjusted index modulation to maximize diffraction efficiency when calculated in the plane-wave approximation. Figure 4(a) shows the maximum diffraction efficiency plotted as a function of the thickness of the intracavity material for two tilt angles ( $\theta_{\mathrm{B}}=0.01$ and 0.1 ) with an incident beam waist of $w_{0}$ $=300 \mu \mathrm{m}$. A threshold thickness appears from which the
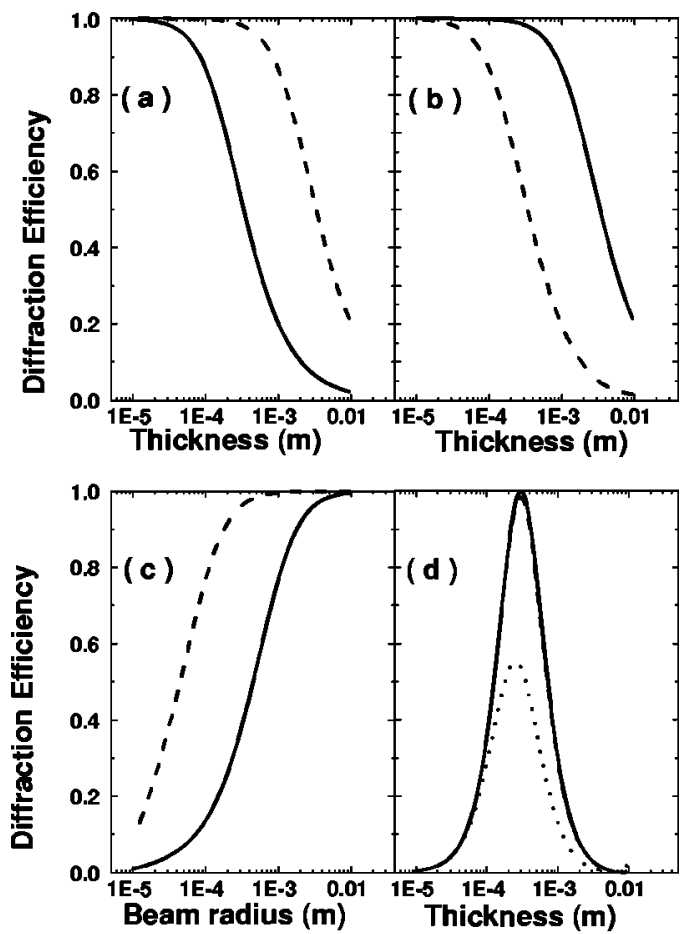

Fig. 4. Diffraction efficiency of the Bragg device under Gaussian illumination (a) versus cavity thickness for various tilt angles (solid curve for $\theta_{\mathrm{B}}=0.1$ and dashed curve for $\theta_{\mathrm{B}}=0.01$ ), (b) versus cavity thickness for various beam waists (solid curve for $w_{0}$ $=300 \mu \mathrm{m}$ and dashed curve for $w_{0}=30 \mu \mathrm{m}$ ), (c) versus beam waist for various tilt angles (solid curve for $\theta_{\mathrm{B}}=0.1$ and dashed curve for $\theta_{\mathrm{B}}=0.01$ ), (d) versus cavity thickness (dotted-dashed curve for a plane wave at $\theta_{\mathrm{B}}=0.1$, solid curve for a beam waist of $w_{0}=300 \mu \mathrm{m}$ at $\theta_{\mathrm{B}}=0.01$ and dotted curve for a beam waist of $w_{0}=300 \mu \mathrm{m}$ at $\theta_{\mathrm{B}}=0.1$. In (a)-(c) the refractive-index modulation is adjusted at every data point for maximum diffraction efficiency in the plane-wave approximation $(\rho=1)$, whereas in (d) the refractive-index modulation is fixed for a maximum value at $l_{0}$ $=300 \mu \mathrm{m}$ in the plane-wave case. diffraction efficiency starts decreasing $\left(l>1 \mathrm{~mm}\right.$ for $\theta_{\mathrm{B}}$ $=0.01$ and $l>100 \mu \mathrm{m}$ for $\theta_{\mathrm{B}}=0.1$ ). This reduction is evidently related to the diffracted beam spread [see Fig. 3(b)] due to the multiple reflections inside the cavity. It must also be stressed that, at least for the small tilt angles considered here, the length threshold of the decrease of the diffraction efficiency is inversely proportional to $\theta_{\mathrm{B}}$, which confirms the geometrical nature of the reduction. The value of this threshold can be related to the finesse of the Fabry-Perot device as follows. If the walk-off $\left(\simeq \mathcal{F} \theta_{\mathrm{B}} l\right)$ of the incident beam due to its multiple reflections in the cavity is greater than the beam diameter, then the cavity is no longer efficient. Figure 4(b) shows the diffraction efficiency of the intracavity grating as a function of the thickness $l$ for two waists of the incident beam $\left(w_{0}\right.$ $=300 \mu \mathrm{m}$ and $\left.w_{0}=30 \mu \mathrm{m}\right)$ and for a tilt angle $\theta_{\mathrm{B}}=0.1$. The curves are very similar to that shown in Fig. 4(a) with a strong decrease of the diffraction efficiency when $l$ $\geqslant w_{0} / \mathcal{F} \theta_{\mathrm{B}}$. The knowledge of this threshold is useful in the design of efficient intracavity Bragg devices. The same behavior can be seen in Fig. 4(c) showing the evolution of the diffraction efficiency of the device as a function of $w_{0}$ for two different tilt angles $\left(\theta_{\mathrm{B}}=0.01\right.$ and 0.1$)$ for a cavity thickness $l_{0}=500 \mu \mathrm{m}$.

The results shown in Figs. 4(a)-4(c) correspond to calculations performed for a refractive-index modulation $\Delta n$ optimized at every data point for a maximum diffraction efficiency in the plane-wave approximation $(\rho=1$ for a lossless intracavity material). However, in a practical device, $\Delta n$ is generally fixed. It is then interesting to examine the behavior of the diffraction efficiency of the device as a function of the various parameters in this case. This is illustrated in Fig. 4(d) showing the diffraction efficiency of the device as a function of the Fabry-Perot thickness in the plane-wave approximation and for a Gaussian incident beam of waist $w_{0}=300 \mu \mathrm{m}$ at two tilt angles $\left(\theta_{\mathrm{B}}\right.$ $=0.01$ and 0.1 ). The index modulation $\Delta n$ is chosen to maximize the diffraction efficiency for a $300-\mu$ m-thick cavity in the plane-wave approximation. For a small tilt angle $\left(\theta_{\mathrm{B}}=0.01\right)$ the results are practically identical for the Gaussian beam and for the plane-wave approximation, even for high thicknesses of the Fabry-Perot cavity for which a decrease of the maximum possible diffraction efficiency was shown [see Fig. 4(a)]. In the case of larger tilt angles $\left(\theta_{\mathrm{B}}=0.1\right)$ the diffraction efficiency at large thicknesses of the Fabry-Perot cavity is smaller than in the case of the plane-wave approximation. Moreover, because of a competition between the Bragg efficiency and the decreasing Fabry-Perot efficiency due to the beam spread, the maximum diffraction efficiency is obtained for a cavity thickness that is slightly smaller than in the plane-wave approximation. This result should be kept in mind when designing an intracavity Bragg device.

\section{ANGULAR SELECTIVITY}

In addition to the decrease in diffraction efficiency, and probably more important, is the weakening of the angular selectivity of intracavity Bragg gratings as a result of the use of small-waist Gaussian beams. Figures 5(a) and 5(b) show the normalized transmission and reflected diffraction efficiency of the device, respectively, plotted as a func- 


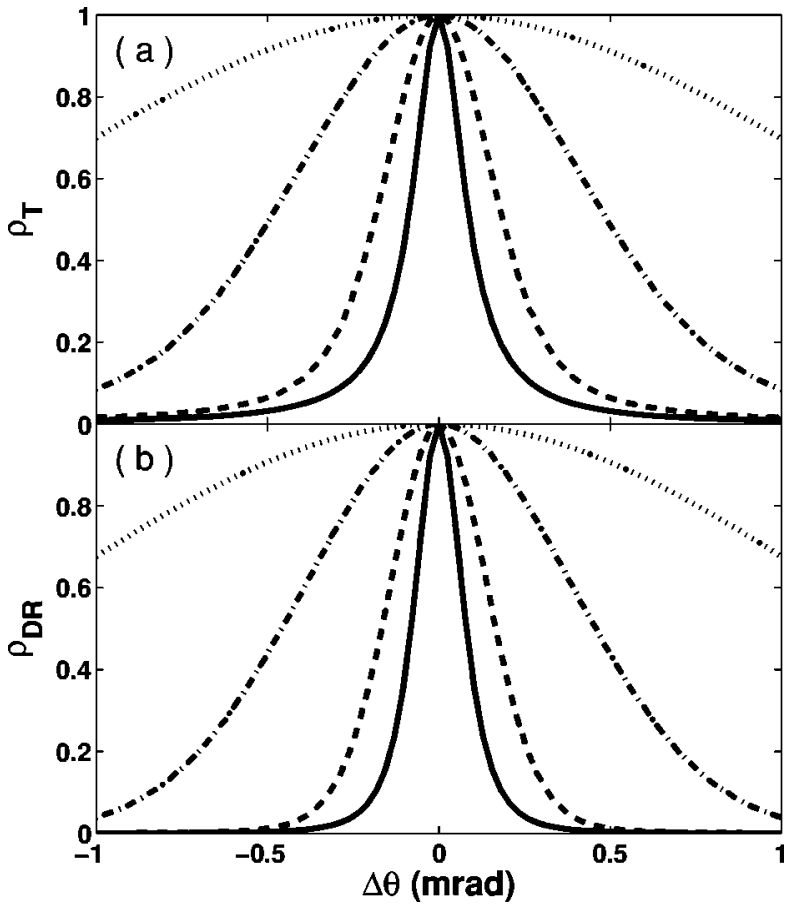

Fig. 5. (a) Normalized transmission $\rho_{\mathrm{T}}$ of the empty FabryPerot cavity and (b) diffraction efficiency $\rho_{\mathrm{DR}}$ of the intracavity Bragg grating plotted as a function of the Bragg detuning for various beam waists (solid, dashed, dotted-dashed and dotted curves for plane waves, $w_{0}=1000,300$, and $100 \mu \mathrm{m}$, respectively). The Fabry-Perot cavity is tuned to the Bragg angle of the grating $\theta_{\mathrm{B}}$

tion of the Bragg detuning $\Delta \theta$. The calculation is performed for plane waves and beam waists of 1000,300 , and $100 \mu \mathrm{m}$ with a nearly totally reflecting $\left(R_{1}=0.8\right.$ and $R_{2}$ $=0.999) 1-\mathrm{mm}$-thick cavity. A $3.8-\mu \mathrm{m}$-period grating $\left(\theta_{\mathrm{B}}\right.$ $=0.1 \mathrm{rad})$ is impressed in a lossless medium $\left(k^{\prime \prime}=0\right)$ of mean refractive index $n_{0}=1.3$. The cavity is tuned to a read beam of mean direction $\theta_{\mathrm{B}}$, and the value of the refractive-index modulation is chosen to obtain a maximum reflected diffraction efficiency for plane waves.

As expected the transmission curves plotted in Fig. 5(a) widen as the beam waist decreases. This is because at small detuning values, some plane-wave components of the Gaussian beam are still resonant, even though the mean direction is not. The maximum values of the transmission and diffraction efficiencies and the corresponding angular FWHM are plotted in Figs. 6(a) and 6(b). Above the beam radius threshold previously mentioned, the behavior tends asymptotically toward that of the plane wave, but the transmission and the diffraction efficiency decrease drastically for decreasing beam waist. For $w_{0}$ $=300 \mu \mathrm{m}$ the FWHM of the reflected diffracted beam $(\approx 1 \mathrm{mrad})$ is increased by a factor of 6 compared to that of the plane wave ( $\approx 0.17 \mathrm{mrad})$. Such results, which demonstrate a low angular selectivity together with a reduced diffraction efficiency, seem to preclude the use of intracavity Bragg gratings for Gaussian read beams of very small waist, at least for thick Fabry-Perot cavities.

This inconvenience can nevertheless be circumvented if the cavity is tuned to the mean incidence angle $\theta=\theta_{\mathrm{B}}$ $+\Delta \theta$ instead of $\theta_{\mathrm{B}}$. This could be done for example by maximizing the transmission of the device. The results of the calculations performed with the same parameters as in Figs. 5 and 6, except for the tuning of the cavity, are presented in Figs. 7 and 8. The transmission is not shown, since the tuning keeps it constant at its maximum value for this cavity. Figure 7 shows the normalized diffraction efficiency $\rho_{\mathrm{DR}} / \rho_{\max }$ plotted as a function of the Bragg detuning $\Delta \theta$ for plane waves and for Gaussian beams with $w_{0}=1000,300$, and $100 \mu \mathrm{m}$. It appears that the angular selectivity is not as drastically reduced as in the case of the fixed Fabry-Perot cavity. This is quantita-

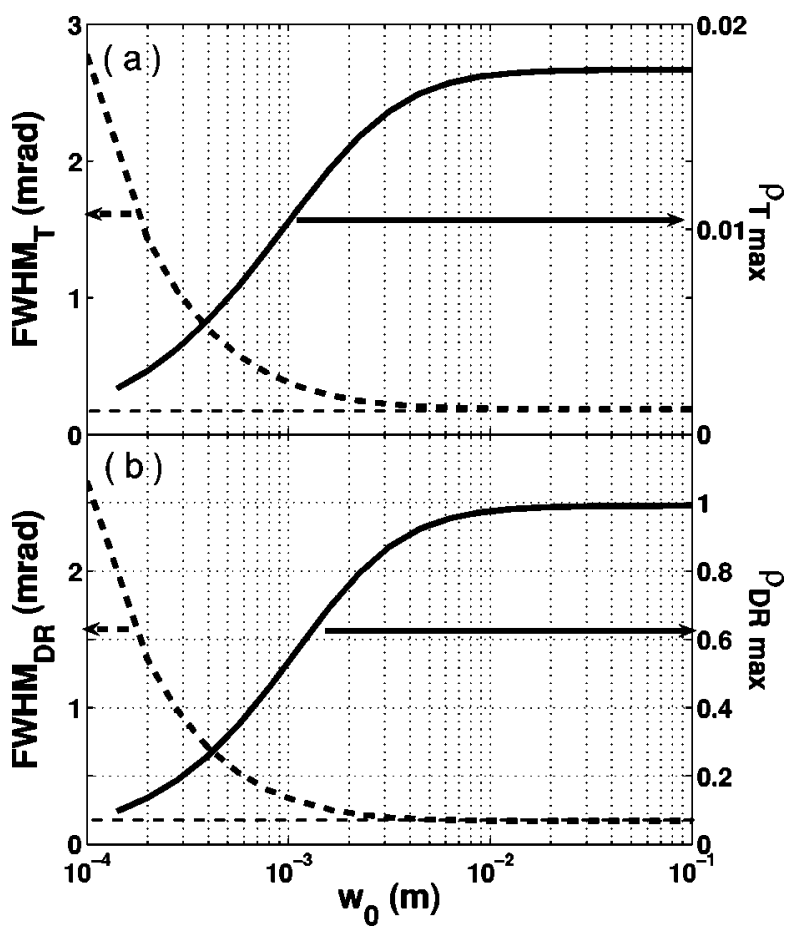

Fig. 6. (a) Maximum transmission $\rho_{\mathrm{T}}$ and angular width FWHM $_{\mathrm{T}}$ of the empty Fabry-Perot cavity and (b) maximum diffraction efficiency $\rho_{\mathrm{DR}}$ and angular width $\mathrm{FWHM}_{\mathrm{DR}}$ of the intracavity Bragg device plotted as a function of the read beam waist; the Fabry-Perot cavity is tuned to the Bragg angle of the grating $\theta_{\mathrm{B}}$.

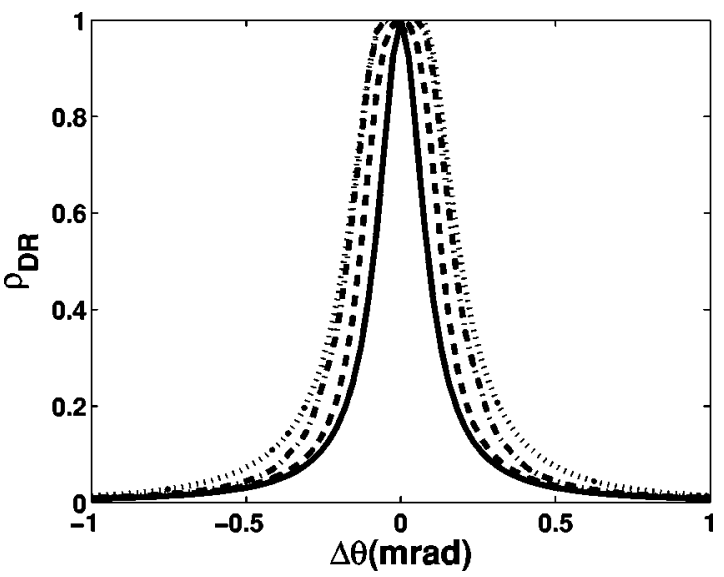

Fig. 7. Normalized diffraction efficiency $\rho_{\mathrm{DR}}$ of the intracavity Bragg grating plotted as a function of the Bragg detuning for various beam waists (solid, dashed, dotted-dashed and dotted curves for plane waves, $w_{0}=1000,300$, and $100 \mu \mathrm{m}$, respectively); the Fabry-Perot cavity is tuned to the incident beam direction $\theta=\theta_{\mathrm{B}}+\Delta \theta$ 


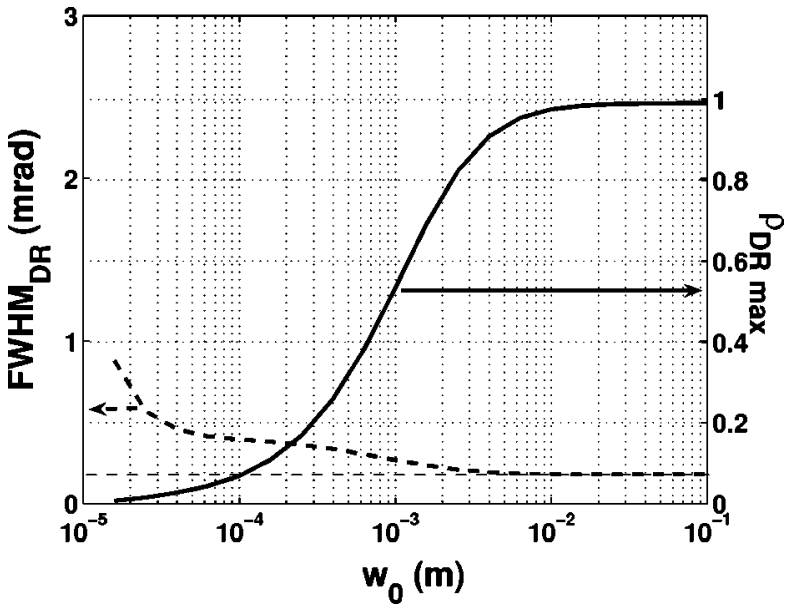

Fig. 8. Maximum diffraction efficiency $\rho_{\mathrm{DR}}$ and angular width $\mathrm{FWHM}_{\mathrm{DR}}$ for the intracavity Bragg device plotted as a function of the read beam waist; the Fabry-Perot cavity is tuned to the incident beam direction $\theta=\theta_{\mathrm{B}}+\Delta \theta$.

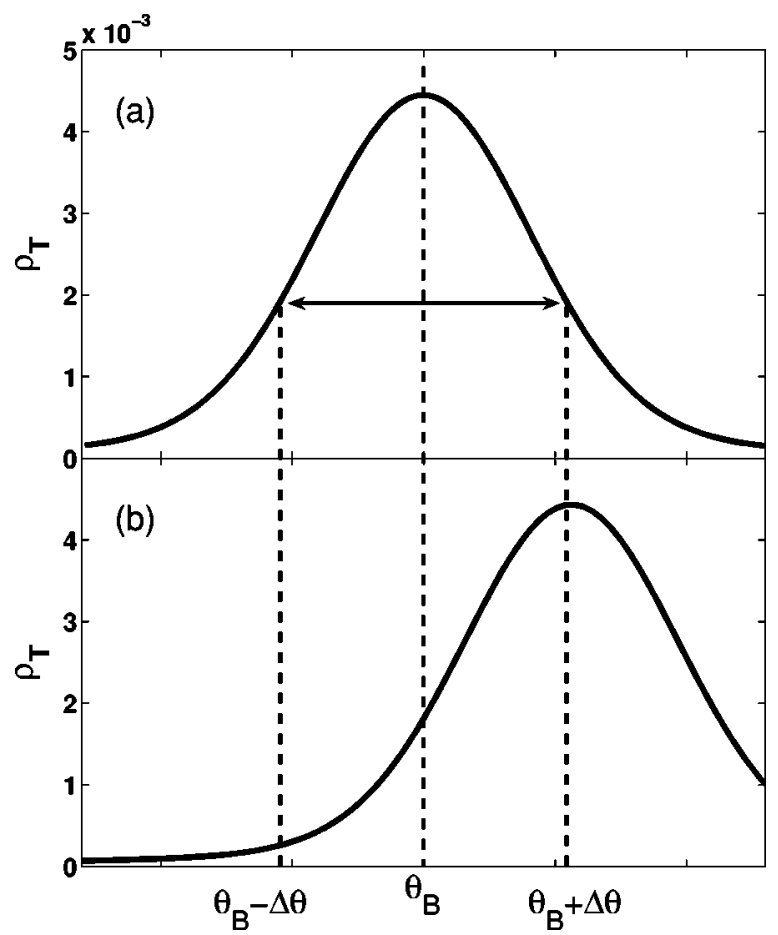

Fig. 9. Normalized transmission $\rho_{\mathrm{T}}$ of the empty Fabry-Perot cavity tuned (a) to the Bragg angle $\theta_{\mathrm{B}}$ and (b) to the read beam direction $\theta_{\mathrm{B}}+\Delta \theta$. The coupling to the Fabry-Perot cavity of the diffracted beam at $\theta_{\mathrm{B}}-\Delta \theta$ is much less in the latter case.

tively confirmed by Fig. 8, which shows the angular FWHM of the reflected diffracted beam plotted as a function of $w_{0}$. Compared with the case of the plane wave (the asymptote), it is multiplied only by 2 for $w_{0}=300 \mu \mathrm{m}$, which demonstrates that intracavity Bragg gratings could be useful for applications to free-space optical parallel processing with reasonable efficiencies $(20 \%$, as also shown in Fig. 8).

It must be stressed that the same performance can be obtained with smaller beam waists and thinner FabryPerot cavities fulfilling the Bragg criterion ${ }^{4}\{2 l[1$ $\left.\left.\left.-R_{1}\right) \sqrt{R_{2}} /\left(1-\sqrt{R_{1} R_{2}}\right)\right]^{2}>10 \Lambda^{2} / \pi \lambda\right\}$ as verified for $w_{0}$
$=10 \mu \mathrm{m}$ and $l=30 \mu \mathrm{m}$, which implies that free-space dense parallel optical processing could also be possible. Concerning applications, the issue of the time response of the device is an important one. The only intrinsic limitation of the intracavity Bragg grating with respect to that parameter is related to the Fabry-Perot lifetime $\tau$ $=\mathcal{F} 2 n l / c$. For $l=30 \mu \mathrm{m}$ and $\mathcal{F}=28$ this lifetime is smaller than 8 ps.

The difference between the results shown in Fig. 6 and those in Fig. 8 can be easily understood if we remember that the beam is diffracted in the direction $-\theta_{\mathrm{B}}+\Delta \theta$ for a read beam sent in the $\theta_{\mathrm{B}}+\Delta \theta$ direction. Indeed, for a cavity tuned to $\theta_{\mathrm{B}}$ and consequently also to $-\theta_{\mathrm{B}}$, the read and diffracted beams are equally coupled to the cavity when $\Delta \theta$ increases [see Fig. 9(a)]. On the other hand, when the cavity is tuned to the read angle $\theta_{\mathrm{B}}+\Delta \theta$ the coupling is drastically reduced for the diffracted beam in the direction $-\theta_{\mathrm{B}}+\Delta \theta$ [see Fig. 9(b)], thus reducing the diffraction efficiency for this incident read beam.

\section{CONCLUSION}

The diffraction of Gaussian beams on intracavity Bragg gratings has been analyzed and compared with that of plane waves. Diffraction efficiency is not significantly altered by the divergence associated with small beam waists. At small Bragg incidence angles it is not affected as long as the walk-off of the incident beam does not exceed the beam diameter and as long as efficient interference can take place in the cavity. The thickness threshold for the decrease of diffraction efficiency is proportional to the tilt angle and inversely proportional to the read beam waist. The angular selectivity of the device is also drastically reduced for a cavity tuned to the Bragg angle of the refractive-index grating. The loss in angular selectivity is nevertheless very small for a Fabry-Perot cavity tuned to the incident beam direction because of the very bad coupling associated with the detuning of the diffracted beam in this case.

This theoretical analysis shows that efficient and freespace dense parallel optical processing can be considered with intracavity Bragg gratings. It should also be stressed that, because of its generality, this calculation can be used to optimize the diffraction properties of any intracavity Bragg grating illuminated by Gaussian beams, which could be useful for any Bragg-diffraction-based optical device.

Corresponding author I. Zaquine's e-mail address is Isabelle.Zaquine@enst.fr.

\section{REFERENCES}

1. R. J. Collier, C. B. Burckhardt, and L. H. Lin, Optical Holography (Academic, Orlando, Fla., 1971).

2. Y. Ding, D. D. Nolte, M. R. Melloch, and A. M. Weiner, "Time-domain image processing using dynamic holography," IEEE J. Sel. Top. Quantum Electron. 4, 332-341 (1998).

3. L. Menez, I. Zaquine, A. Maruani, and R. Frey, "Intracavity Bragg gratings," J. Opt. Soc. Am. B 16, 1849-1855 (1999).

4. L. Menez, I. Zaquine, A. Maruani, and R. Frey, "Bragg thickness criterion for intracavity diffraction gratings," J. Opt. Soc. Am. B 19, 965-972 (2002). 
5. L. Menez, I. Zaquine, A. Maruani, and R. Frey, "Intracavity refractive index Bragg gratings in absorbing and amplifying media," Opt. Commun. 204, 267-275 (2002).

6. L. Menez, I. Zaquine, A. Maruani, and R. Frey, "Experimental investigation of intracavity Bragg gratings," Opt. Lett. 27, 479-481 (2002).

7. K. Sundar, N. Mukunda, and R. Simon, "Coherent-mode decomposition of general anisotropic Gaussian Schellmodel beams," J. Opt. Soc. Am. A 12, 560-569 (1995).

8. X. Xue, H. Wei, and A. G. Kirk, "Intensity-based modal decomposition of optical beams in terms of HermiteGaussian functions,” J. Opt. Soc. Am. A 17, 1086-1091 (2000).

9. J.-S. Lee and C.-S. Shim, "Characteristics of a spectrumslicing filter composed of an angle-tuned Fabry-Perot etalon and a Gaussian input beam," IEEE Photonics Technol. Lett. 7, 905-907 (1995).
10. P. L. Penna, A. D. Virgilio, M. Fiorentino, A. Porzio, and S. Solimeno, "Transmittivity profile of high finesse plane parallel Fabry-Perot cavities illuminated by Gaussian beams," Opt. Commun. 162, 267-279 (1999).

11. O. Mata-Mendez and F. Chavez-Rivas, "Diffraction of Gaussian and Hermite-Gaussian beams by finite gratings," J. Opt. Soc. Am. A 18, 537-545 (2001).

12. S.-D. Wu and E. N. Glytsis, "Finite-number-of-periods holographic gratings with finite-width incident beams: analysis using the finite-difference frequency-domain method," J. Opt. Soc. Am. A 19, 2018-2029 (2002).

13. T. K. Gaylord and M. G. Moharam, "Planar dielectric grating diffraction theories," Appl. Phys. B 28, 1-14 (1982)

14. R. E. Collin, Field Theory of Guided Waves (Mc Graw-Hill, New York, 1960), p. 368.

15. R. W. Boyd, Nonlinear Optics (Academic, San Diego, Calif. 2003), chap. 2, p. 73. 